\title{
BMJ Global Health Redefining typhoid diagnosis: what would an improved test need to look like?
}

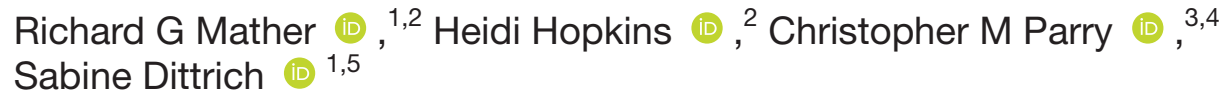

To cite: Mather RG, Hopkins $\mathrm{H}$, Parry CM, et al. Redefining typhoid diagnosis: what would an improved test need to look like? BMJ Global Health 2019;4:e001831. doi:10.1136/ bmjgh-2019-001831

Handling editor Seye Abimbola

- Additional material is published online only. To view please visit the journal online (http://dx.doi.org/10.1136/ bmjgh-2019-001831).

Received 10 July 2019 Revised 4 October 2019 Accepted 5 October 2019

Check for updates

(c) Author(s) (or their employer(s)) 2019. Re-use permitted under CC BY-NC. No commercial re-use. See rights and permissions. Published by BMJ.

${ }^{1}$ Malaria and Fever Program, Foundation for Innovative New Diagnostics (FIND), Geneva, Switzerland

${ }^{2}$ Faculty of Infectious and Tropical Diseases, London School of Hygiene \& Tropical Medicine, London, UK ${ }^{3}$ Department of Clinical Sciences, Liverpool School of Tropical Medicine, Liverpool, UK ${ }^{4}$ School of Tropical Medicine and Global Health, Nagasak University, Nagasaki, Japan ${ }^{5}$ Nuffield Department of Medicine, University of Oxford, Oxford, UK

Correspondence to Dr Sabine Dittrich; sabine.dittrich@finddx.org

\section{ABSTRACT}

Introduction Typhoid fever is one of the most common bacterial causes of acute febrile illness in the developing world, with an estimated 10.9 million new cases and 116.8 thousand deaths in 2017. Typhoid point-of-care (POC) diagnostic tests are widely used but have poor sensitivity and specificity, resulting in antibiotic overuse that has led to the emergence and spread of multidrug-resistant strains. With recent advances in typhoid surveillance and detection, this is the ideal time to produce a target product profile (TPP) that guides product development and ensure that a next-generation test meets the needs of users in the resource-limited settings where typhoid is endemic. to develop a draft TPP for a next-generation typhoid diagnostic test with minimal and optimal desired characteristics for 36 test parameters. The TPP was refined using feedback collected from a Delphi survey of key stakeholders in clinical medicine, microbiology, diagnostics and public and global health.

Results A next-generation typhoid diagnostic test should improve patient management through the diagnosis and treatment of infection with acute Salmonella enterica serovars Typhi or Paratyphi with a sensitivity $\geq 90 \%$ and specificity $\geq 95 \%$. The test would ideally be used at the lowest level of the healthcare system in settings without a reliable power or water supply and provide results in $<15$ min at a cost of $<$ US $\$ 1.00$.

Conclusion This report outlines the first comprehensive TPP for typhoid fever and is intended to guide the development of a next-generation typhoid diagnostic test. An accurate POC test will reduce the morbidity and mortality of typhoid fever through rapid diagnosis and treatment and will have the greatest impact in reducing antimicrobial resistance if it is combined with diagnostics for other causes of acute febrile illness in a treatment algorithm.

\section{INTRODUCTION}

Typhoid fever (typhoid) is an enteric bacterial infection caused by Salmonella enterica serovar Typhi (Salmonella Typhi; S. Typhi). It is one of the most common bacterial causes of acute febrile illness in the developing world, ${ }^{1}$ with an estimated 10.9 million new Methods A structured literature review was conducted

\section{Key questions}

What is already known?

- Current diagnostic tests for typhoid fever lack the sensitivity and specificity to be reliably used at the point-of-care (POC), resulting in the overuse of antibiotics through empiric treatment.

What are the new findings?

- To meet the needs of users in endemic regions, stakeholders proposed that an improved typhoid diagnostic test must be able to detect $S$. Paratyphi as well as $S$. Typhi, have a sensitivity $\geq 90 \%$, a specificity $\geq 95 \%$ and have an end user cost of $<$ US $\$ 3$.

What do the new findings imply?

- To have a meaningful impact on the overuse of antibiotics that has contributed to the emergence of antibiotic resistance in $S$. Typhi and other bacteria, an improved typhoid POC test needs to be used as part of a treatment algorithm in conjunction with diagnostics for malaria and other causes of acute febrile illness.

cases worldwide and 116.8 thousand deaths in 2017. ${ }^{23}$ Paratyphoid fever caused by Salmonella enterica serovars Paratyphi A, B and C ( $S$. Paratyphi) results in a disease that can have an identical clinical syndrome to typhoid fever, ${ }^{4}$ but is often less severe. ${ }^{5}$ Typhoid fever is most common in South Asia and sub-Saharan Africa, with children predominantly affected. Like many febrile illnesses, typhoid presents with non-specific symptoms and signs, especially in its early stages. In routine healthcare settings in low/middleincome countries (LMICs), typhoid fever is commonly suspected and treated empirically with antibiotics. ${ }^{6}$ This overuse of antibiotics creates a selective pressure for the development of antimicrobial resistance (AMR), ${ }^{7}$ that has resulted in the emergence and spread of typhoid strains that are resistant to all firstline antibiotics. ${ }^{8}$ Similarly, the low specificity of current rapid diagnostic tests (RDTs) can 
lead to an over diagnosis of typhoid fever that may result in the overuse of antibiotics and delay the proper treatment for underlying conditions. For example, the use of the Widal test during an outbreak of acute febrile illness in Nepal led to misdiagnosis of typhoid which delayed the appropriate treatment of the causative agent (scrub typhus), resulting in dozens of deaths. ${ }^{9}$ The potential harms of current typhoid RDTs are compounded by the fact they are widely available, cheap and easy to use. ${ }^{10}$

Various aspects of $S$. Typhi biology make diagnosis by standard laboratory methods challenging. $S$. Typhi is able to bypass the gastrointestinal mucosal barrier that restricts other enteric bacteria and can evade the typical innate immune responses with limited activation of inflammatory pathways. ${ }^{11} S$. Typhi infection begins with invasion of the mucosa of the terminal ileum, and the organism is thought to only be transiently present in the blood before dissemination throughout the reticuloendothelial system into the bone marrow, liver and spleen. ${ }^{12}$ The bacterial load in peripheral blood peaks in the first week of illness, ${ }^{13}$ but is still very low with a median of $0.1-1.0$ colony-forming units $/ \mathrm{mL}$ in symptomatic patients. ${ }^{14}$ This concentration is difficult to detect by blood culture or PCR, resulting in lower sensitivity for these diagnostic tests. $S$. Typhi is a member of the Enterobacteriaceae family, and antibodies that have been produced in response to prior infections with other Enterobacteriaceae tend to cross-react with $S$. Typhi, ${ }^{12}$ due to significant conservation of surface antigens. This cross-reactivity lowers the specificity of antibody-based diagnostic assays that otherwise are well suited for a simple, rapid and inexpensive test format. Compounding the challenge, the muted immune response that occurs through expression of the Vi capsular polysaccharide ${ }^{12}$ may further hinder the utility of serological tests for typhoid diagnosis.

In clinical settings supported by a microbiology laboratory, invasive typhoid infection is confirmed through isolation of $S$. Typhi from blood cultures, but this is relatively expensive, can take $>48$ hours, has low sensitivity and requires laboratory infrastructure and trained staff that are not commonly available in LMIC where typhoid is most prevalent. ${ }^{15}$ Bone marrow cultures have high sensitivity for detection of $S$. Typhi but are not routinely used because of the invasive techniques needed to obtain bone marrow aspirates. PCR testing for typhoid is expensive and has a low diagnostic sensitivity when used on peripheral blood samples. ${ }^{16}$ Other available pointof-care (POC) diagnostic tests include the Widal test, ${ }^{17}$ TUBEX, ${ }^{18}$ Typhidot ${ }^{19}$ Test-it Typhoid ${ }^{20}$ and the TyphoidParatyphoid diagnostic assay (TPTest). However, these tests all have significant drawbacks that limit their clinical use.

Serological tests are limited by low antibody titers in the early stage of infection and may produce falsenegative results for IgM-based assays depending on the timing of testing. ${ }^{21}$ The Widal test is a quantitative agglutination test that identifies serum antibodies against Salmonella antigens $\mathrm{O}$-somatic and $\mathrm{H}$-flagellar. The test is considered positive if a convalescent phase serum sample has a fourfold higher titer than an acute sample. But the Widal test is often applied incorrectly with just a single acute phase sample used for diagnosis and treatment. ${ }^{22}$ A Cochrane review of the accuracy of the commercially available antibody-based rapid RDTs showed moderate sensitivity and specificity for the TUBEX colorimetric test that detects anti-O:9 antibody titres $(78 \%, 87 \%)$, the Typhidot dot ELISA that measures $\operatorname{IgG}$ and $\operatorname{IgM}$ antibodies against the outer membrane proteins of $S$. Typhi $(84 \%, 79 \%)$ and the Test-it Typhoid immunochromatographic lateral flow assay that detects $\mathrm{IgM}$ antibodies against $S$. Typhi O antigen $(69 \%, 90 \%) .{ }^{23}$ The TPTest is a newer serological test that detects circulating IgA using ELISA with a sensitivity and specificity of $>95 \% .{ }^{24}$ But this assay is not commercially available, takes $24-48$ hours to produce a result, and requires isolation and culturing of peripheral blood mononuclear cells which is technically challenging, utilising equipment not widely available in resource-limited settings. ${ }^{1424}$ Due to the limited sensitivity of all current typhoid POC tests they cannot be relied on to guide treatment prescribing.

If developed and implemented effectively, an accurate typhoid RDT could reduce morbidity and mortality through faster diagnosis. Further, it could help to reduce the overuse of antibiotics that contributes to the emergence and spread of multidrug-resistant strains of $S$. Typhi and other bacteria. In recent years, novel approaches have been described to develop typhoid diagnostic tests with improved accuracy in resource-limited settings, including serological, molecular, metabolomic, proteomic and transcriptomic methods. ${ }^{7}$ For example, a recent study has shown that IgA and IgM against $S$. Typhi lipopolysaccharide may be a specific marker of acute typhoid infection and is a promising target for diagnostic test development. ${ }^{25}$

As the need for appropriate fever case management becomes more apparent,${ }^{26}$ the need for improved typhoid diagnostics suitable for use in low-resource environments becomes more pressing. Building on the recent momentum around improved typhoid surveillance ${ }^{27}$ and advances in typhoid detection, ${ }^{1}$ this report describes the development of a target product profile (TPP) in an attempt to define the diagnostic needs for this important pathogen. The TPP is intended to guide product development and to ensure an optimised solution that meets the needs of endemic countries and results in tangible improvements in patient management. In addition, the TPP aims to reinvigorate the discussion of diagnostics as a crucial part of the global typhoid agenda. This report focuses on the process of TPP development with an emphasis on key test characteristics and discussion points identified by typhoid experts and experienced stakeholders.

\section{METHODS \\ Data gathering}

A structured review of relevant literature related to Salmonella Typhi diagnosis was performed to develop a draft 


\begin{tabular}{ll}
\hline Table 1 Typhoid & target product profile parameters \\
\hline Category & Test characteristic \\
\hline Scope of test & Goal \\
& Target population \\
& Target user \\
& Target level of health system \\
Test performance & Sample type \\
& Sample collection \\
& Sample volume/ sample transfer device \\
& Additional sample preparation \\
& Ease of use \\
& Hands on time \\
& Time to result \\
& Read out of results \\
& In use stability \\
& Data output +connectivity \\
& Data interpretation \\
& Analyte type \\
& Multiplexing \\
& Analytical sensitivity/limit of detection \\
Diagnostic sensitivity \\
Diagnostic specificity \\
Reproducibility \\
Kit configuration \\
Reagent preparation \\
Operating conditions \\
Transportation and storage stability \\
Equipment (instrumentation external to \\
test) \\
External maintenance \\
Calibration \\
Internal/ process control \\
Batch/quality control \\
Power requirements \\
Water requirement \\
Waste disposal \\
Tio-safety \\
Cost per test \\
\hline Operational \\
characteristics
\end{tabular}

TPP with minimal and optimal desired characteristics for a next-generation typhoid diagnostic test. The test characteristics chosen for the TPP were selected based on previous TPPs published by the Foundation for Innovative New Diagnostics (FIND), ${ }^{28} 29$ and include the scope, target population, intended use, expected test performance, as well as operational and financial parameters (table 1). Each desired test characteristic was classified as either a minimum requirement that a test must meet to be useful for healthcare providers treating patients in resource-limited settings, or an optimum threshold that would make the test highly desirable for both healthcare providers and patients. The Ovid Medline database was accessed on 21 June 2018 using the Medical Subject Heading (MeSH) 'Typhoid fever' and the subheading 'Diagnosis'. Results were restricted to English language articles published in the previous 10 years. Titles and abstracts of retrieved articles were scanned for relevance, with articles of interest thoroughly reviewed by RGM for content relevant to the TPP. Additional documents were identified by searching for 'typhoid' on the websites of the Cochrane library, WHO and FIND, and by screening references and studies that cited articles selected in the initial search. Expert stakeholders to be contacted for the Delphi survey were identified as part of the literature review.

\section{Delphi survey}

Stakeholders were contacted for input on the draft TPP using a Delphi survey. Stakeholders included specialists in clinical medicine $(n=14)$, laboratory medicine $(\mathrm{n}=2)$, microbiology $(\mathrm{n}=6)$, diagnostics $(\mathrm{n}=11)$ and public health and global health $(n=6)$. An online survey (online supplementary file 1) was used and respondents were asked to rate their agreement with each of the TPP characteristics using a Likert scale $(1=$ strongly disagree, $2=$ disagree, $3=$ neither agree nor disagree, $4=$ mostly agree, $5=$ fully agree). A consensus agreement was defined as $\geq 75 \%$ of respondents who either mostly or fully agreed with a TPP characteristic. Results from the first round of the survey (October 2018) were used to refine the TPP, and a second draft of the TPP was distributed (November 2018) to all initial participants as well as two additional stakeholders identified after the first round of the survey was completed.

\section{Patient and public involvement}

While key stakeholders were involved in developing the TPP, there was no patient or public involvement in this research.

\section{RESULTS}

An Ovid Medline search using the MeSH Typhoid fever produced 10698 results, with 1558 results for the subheading Diagnosis. Limiting search results to English language articles published after 1 January 2008, provided 298 articles that were screened for relevance. Additional documents were included as outlined in the Methods section and selected articles were thoroughly reviewed to develop a draft typhoid TPP with minimum and optimum criteria for the test characteristics in table 1 .

Feedback on the draft TPP was obtained from key stakeholders through the first round of the Delphi survey, with 40 stakeholders contacted and 19 (19/40, 48\%) completed surveys received. Survey respondents reported having experience working in low-resource settings in Africa $(n=14)$, the Americas $(n=2)$, Europe $(n=2)$, the Eastern Mediterranean $(n=2)$, South-East Asia $(n=12)$ and the Western Pacific region $(n=2)$, with multiple respondents reporting experience in more than one region. Consensus agreement of $\geq 75 \%$ was achieved for $34 / 36(94 \%)$ TPP criteria. TPP criteria that generated the most discussion in the Delphi survey were related to the scope of the test including the goal, target population, level of the healthcare system, diagnostic sensitivity and specificity, as well as cost. Based on feedback from survey respondents, 'multiplexing' was removed as a TPP characteristic, and the remaining minimum and optimum TPP criteria were revised. For criteria that had achieved consensus agreement, revisions 
Table 2 Typhoid target product profile characteristics: scope of the test

\begin{tabular}{llll}
\hline Characteristic & Minimal requirement & Optimal requirement & References \\
\hline Goal & $\begin{array}{l}\text { Point-of-care test to improve patient } \\
\text { management through diagnosis and } \\
\text { treatment of infection with acute } \\
\text { Salmonella enterica serovars Typhi or } \\
\text { Paratyphi }\end{array}$ & $\begin{array}{l}\text { Combine with diagnostics for } \\
\text { malaria and other causes of acute } \\
\text { febrile illness as part of a treatment } \\
\text { algorithm }\end{array}$ \\
Target population & All individuals with undifferentiated acute fever & Healthcare worker & 152730 \\
Target user & Laboratory technician & Primary health posts and centres & 631 \\
Target level of health system* & District hospital with basic laboratory \\
facilities & & & \\
\hline
\end{tabular}

${ }^{*}$ Consensus not reached among survey respondents.

were made if survey respondents provided compelling suggestions for improvement.

A second draft of the TPP was distributed to the 19 people who responded to the initial Delphi survey, and two additional stakeholders identified after completion of the first round. A total of 12 completed surveys were received from 13 stakeholders, including two who submitted a joint survey, with consensus agreement achieved for 33/35 (94\%) TPP characteristics. The two criteria that did not meet the consensus threshold were the target level of the health system and diagnostic sensitivity, both of which received $67 \%$ agreement. Survey respondent feedback was used to revise these two criteria, and to make minor changes to four criteria that had $\geq 75 \%$ agreement, before inclusion in the final version of the TPP presented in tables 2-4.

\section{Scope of test}

Delphi survey feedback emphasised that a nextgeneration RDT for typhoid fever should not focus solely

Table 3 Test performance characteristics for a typhoid diagnostic target product profile

\begin{tabular}{|c|c|c|c|}
\hline Characteristic & Minimum requirement & Optimum requirement & References \\
\hline Sample type & Venous blood & $\begin{array}{l}\text { Capillary blood or less invasive sample } \\
\text { type (eg, saliva), excluding stool and } \\
\text { urine }\end{array}$ & 1228 \\
\hline Sample collection & \multicolumn{2}{|c|}{ Transfer and quantification device included in the test } & Industry standard \\
\hline $\begin{array}{l}\text { Sample volume/ } \\
\text { sample transfer device }\end{array}$ & $\leq 1 \mathrm{~mL}$ venous blood & $\leq 100 \mu \mathrm{L}$ capillary blood & 47 \\
\hline $\begin{array}{l}\text { Additional sample } \\
\text { preparation }\end{array}$ & 2 sample processing steps & None required & $\begin{array}{l}\text { May need to separate } \\
\text { serum from whole blood } \\
\text { before applying to RDT }\end{array}$ \\
\hline Ease of use & $\leq 5$ steps, of which $\leq 2$ are timed & $\leq 3$ steps, of which $\leq 1$ is timed & 6 \\
\hline Total hands-on time & $\leq 5 \min$ & $\leq 1 \mathrm{~min}$ & 728 \\
\hline Time to result & $\leq 2$ hours & $\leq 15 \mathrm{~min}$ & 2834 \\
\hline Read out of results & Binary results & & 6 \\
\hline In use stability & Results stable $\geq 15 \mathrm{~min}$ & Results stable $\geq 1$ hour & 28 \\
\hline $\begin{array}{l}\text { Data output and } \\
\text { connectivity }\end{array}$ & No wireless connectivity required & $\begin{array}{l}\text { Wireless connectivity used to transfer } \\
\text { data }\end{array}$ & 48 \\
\hline Data interpretation & $\begin{array}{l}\text { Readout easily interpretable by } \\
\text { non-laboratory personnel }\end{array}$ & No data interpretation required & 7 \\
\hline Analyte type & $\begin{array}{l}\text { Any acceptable analyte, or combir } \\
\text { sensitivity and specificity thresholc }\end{array}$ & $\begin{array}{l}\text { lation of analytes, that can meet } \\
\text { is }\end{array}$ & 7 \\
\hline $\begin{array}{l}\text { Analytical sensitivity/ } \\
\text { limit of detection }\end{array}$ & $\begin{array}{l}\text { Limit of detection should be such } \\
\text { performance as defined below }\end{array}$ & that it allows clinically relevant & 7 \\
\hline Diagnostic sensitivity* & $\geq 90 \%$ & $\geq 95 \%$ & 2223 35-37 \\
\hline Diagnostic specificity & $\geq 95 \%$ & $\geq 98 \%$ & $222335-37$ \\
\hline Reproducibility & Kappa $\geq 0.9$ between different ope & rators and laboratories/locations & 49 \\
\hline
\end{tabular}

${ }^{*}$ Consensus not reached among survey respondents. 
Table 4 Consensus operational characteristics for the typhoid target product profile

\begin{tabular}{|c|c|c|c|}
\hline Characteristic & Minimum requirement & Optimum requirement & References \\
\hline Kit configuration & $\begin{array}{l}\text { Package of single kits sharing reagents (if } \\
\text { required) and user manual. Instructions in } \\
\text { English, French, Spanish and Portuguese }\end{array}$ & $\begin{array}{l}\text { Package of single kits with } \\
\text { individual reagents (if required) } \\
\text { sharing user manual. Instructions in } \\
\text { local languages }\end{array}$ & 2838 \\
\hline Reagent preparation & One reagent preparation step & None required & 2839 \\
\hline Operating conditions & $\begin{array}{l}- \text { Between } 5^{\circ} \mathrm{C} \text { and } 40^{\circ} \mathrm{C} \\
\leq 90 \% \text { relative humidity }\end{array}$ & $\begin{array}{l}\text { Between } 5^{\circ} \mathrm{C} \text { and } 45^{\circ} \mathrm{C} \\
\leq 90 \% \text { relative humidity }\end{array}$ & 38 \\
\hline $\begin{array}{l}\text { Transportation and } \\
\text { storage stability }\end{array}$ & $\begin{array}{l}\geq 12 \text { months at } \leq 35^{\circ} \mathrm{C} \text { and } \leq 70 \% \text { relative } \\
\text { humidity, no cold chain needed, ability } \\
\text { to withstand transport stress ( } \leq 3 \text { days at } \\
60^{\circ} \mathrm{C} \text { ) }\end{array}$ & $\begin{array}{l}\geq 24 \text { months at } \leq 45^{\circ} \mathrm{C} \text { and } \leq 90 \% \\
\text { relative humidity, no cold chain } \\
\text { needed, ability to withstand } \\
\text { transport stress ( } \leq 3 \text { days at } 60^{\circ} \mathrm{C} \text { ) }\end{array}$ & 38 \\
\hline $\begin{array}{l}\text { Equipment } \\
\text { (instrumentation external } \\
\text { to test) }\end{array}$ & $\begin{array}{l}\text { Small, portable or handheld, battery- } \\
\text { operated instrument }\end{array}$ & No equipment & 67 \\
\hline External maintenance & $\begin{array}{l}\text { Minimal maintenance, simple to perform } \\
\text { by non-laboratory personnel }\end{array}$ & No maintenance & 28 \\
\hline Calibration & $\begin{array}{l}\leq 1 \text { annual calibration, ideally } \\
\text { autocalibration by operator or remotely }\end{array}$ & No calibration & 28 \\
\hline Internal/ process control & Included in each assay & & Industry standard \\
\hline Batch/quality control & \multicolumn{2}{|c|}{ Positive and negative controls included in each kit } & Industry standard \\
\hline Power requirements & Battery or solar powered & No power needed & 28 \\
\hline Water requirement & No external water required & & 28 \\
\hline Waste disposal & Biohazard waste, sharps disposal & $\begin{array}{l}\text { No toxic waste requiring special } \\
\text { disposal }\end{array}$ & 50 \\
\hline Bio-safety & $\begin{array}{l}\text { Basic biosafety level 1, WHO Class B In- } \\
\text { vitro diagnostic (moderate individual and } \\
\text { low public health risk) }\end{array}$ & $\begin{array}{l}\text { Basic biosafety level 1, WHO Class } \\
\text { A In-vitro diagnostic (low individual } \\
\text { and low public health risk). }\end{array}$ & 5051 \\
\hline Training requirements & $\leq 0.5$ days for lab technician & $\begin{array}{l}\leq 0.5 \text { days for experienced health } \\
\text { worker }\end{array}$ & 6723 \\
\hline Cost per test & End-user cost $<$ US $\$ 3.00$ & End-user cost $<$ US $\$ 1.00$ & 728 \\
\hline
\end{tabular}

on the diagnosis of $S$. Typhi. To reduce the empiric use of antibiotics that generates selective pressure for AMR, a typhoid RDT would ideally be combined with diagnostics for malaria and other causes of acute febrile illness as part of a case management algorithm (table 2). Due to the similar clinical presentation and the changing epidemiology of $S$. Typhi and $S$. Paratyphi, survey respondents advised that a next-generation test for typhoid fever should be able to detect both $S$. Typhi and $S$. Paratyphi.

The target population was identified based on published data from Africa and Asia. ${ }^{27}$ Children aged 2-14 years bear the brunt of the global typhoid burden but there is substantial variability both within and between regions in terms of who is most affected. A recent study from Pakistan found higher rates of typhoid fever in adults than in children. ${ }^{30}$ These data highlight the need for a typhoid RDT that can detect the disease in individuals of all ages, as confirmed by the Delphi survey. A next-generation typhoid POC test should be able to accurately diagnose typhoid in all individuals with undifferentiated acute fever at any point in their disease course from the onset of fever until the time they present for care.
When designing new diagnostic tests, the level of the healthcare system where a test will be deployed is an important consideration. Ghani et al have identified five healthcare system levels, with different types of diagnostic or prognostic tools suitable for different levels. ${ }^{31}$ Typhoid is most prevalent in LMIC with limited healthcare resources, and in these contexts the optimal typhoid test would not require sophisticated equipment and could be easily interpreted by non-laboratory personnel. ${ }^{6} 7$ Respondents agreed that a test would optimally be usable at the lowest level of a healthcare system, which in many cases is a community health worker seeing patients in an informal environment. However, as the current gold standard of blood culture requires laboratory equipment, but has suboptimal sensitivity and specificity, some respondents felt it was acceptable for a typhoid RDT to require basic laboratory facilities, with a trained laboratory technician, providing it meets all other TPP criteria. Based on feedback from the initial round of the Delphi survey the minimum target level was adjusted upward to a higher level of the healthcare system, but consensus agreement was not achieved as some respondents felt strongly that 
the minimum requirement should be a test that can be used in informal settings at the lowest level of the healthcare system.

\section{Test performance}

Blood culture is commonly used as the reference standard for typhoid diagnosis but requires sophisticated equipment not readily available in LMIC where typhoid is endemic. ${ }^{6}$ Typhoid blood cultures require a minimum of $2-10 \mathrm{~mL}$ of venous blood due to the low bacterial load in peripheral blood, and have poor sensitivity estimated at only $61 \%$ in a recent systematic review. ${ }^{32}$ A composite reference standard (CRS) that combines multiple tests with high specificity but suboptimal sensitivity has been proposed as a possible way to improve diagnostic accuracy. ${ }^{733}$ Various test combinations have been used as a CRS for typhoid, ${ }^{33}$ but respondents in this Delphi exercise advised that the adoption of a standardised composite is required before it can be included in a TPP.

The most commonly used typhoid POC tests (Widal, Typhidot, Tubex, Test-It Typhoid, TPTest) require between $5 \mu \mathrm{L}$ and $1 \mathrm{~mL}$ of blood, but have only moderate sensitivity and specificity. ${ }^{23}$ Survey respondents agreed that an optimal next-generation typhoid RDT would use a capillary blood sample with a volume of $\leq 100 \mu \mathrm{L}$, or a less invasive sample type, excluding urine and stool. $^{12}{ }^{28}$ However, survey respondents indicated that $\leq 1 \mathrm{~mL}$ of venous blood was an acceptable minimum requirement due to the current difficulty in accurately diagnosing typhoid fever (table 3). The TPP allows for up to two sample processing steps as an RDT may require serum to be separated from whole blood, with at most five steps for the test of which no more than two should be timed, ${ }^{28}$ and a total hands on time of $<5 \mathrm{~min}$. Based on a published expert consensus TPP for diagnostics for acute febrile illness, RDT performance ideally would entail three or fewer steps, of which at most one step is timed, with a total hands-on time of 1 min or less. ${ }^{28}$

For a new typhoid diagnostic test to have the greatest impact on prescribing and clinical outcomes it would need to yield results in less than a few hours. ${ }^{34}$ There was consensus agreement that the optimum requirement would make results available within $15 \mathrm{~min}$ to coincide with the average development time of other POC diagnostics commonly used in LMIC environments (for example, malaria antigen-detecting RDTs). A minimum requirement of results within 2 hours was agreed; this would be a significant improvement from the $\geq 48$ hours required for blood culture, and 2 hours was deemed the longest time that outpatients could wait for test results, particularly in rural settings where patients may have to travel long distances to reach a health facility. ${ }^{28}$

Researchers have proposed that an ideal typhoid diagnostic test would have a simple positive/negative read-out similar to a home pregnancy test, ${ }^{6}$ with results easily interpretable by non-laboratory personnel. ${ }^{7}$ The typhoid TPP therefore requires a binary read-out of results, with data that either do not require interpretation, or that are easily interpretable. No specific analyte or limit of detection is specified for the typhoid TPP, with any analyte or combination of analytes acceptable providing the test meets all other TPP requirements.

The minimum TPP requirement for diagnostic sensitivity is $\geq 90 \%$, with an optimum sensitivity of $\geq 95 \%$, based on modelling data and expert opinion. ${ }^{222335-37}$ Consensus agreement in the Delphi survey was not achieved for test sensitivity, which reflects the substantial variation in published expert opinions regarding the desirable accuracy for a typhoid RDT. However, survey respondents did agree on a minimum specificity of $95 \%$ and an optimum specificity of $98 \%$.

\section{Operational characteristics}

Survey respondents agreed on operational characteristics of the typhoid TPP (table 4). Typhoid diagnostic test kits ideally should consist of individually packaged tests with individual reagents (if required) and a user manual in local languages, based on TPP characteristics for other POC tests in regions where typhoid is endemic. ${ }^{28} 3839 \mathrm{Up}$ to one reagent preparation step is acceptable, to allow for reconstitution of a powdered reagent. The test should not require a cold chain, with operating conditions that reflect the high temperatures and humidity that are present in many regions in Africa and Asia where typhoid is prevalent.

Currently, most typhoid treatments are provided in outpatient settings, including informal medical shops, so an ideal POC test would not require any sophisticated equipment or a formal laboratory infrastructure. ${ }^{7}$ A small, portable or handheld battery-operated instrument is acceptable,${ }^{40}$ but ideally no equipment would be required. To be truly transformative, a typhoid POC test needs to be useable in settings without a reliable power or water supply. If power is required, then it should be provided by a combination of rechargeable batteries and solar power.

Empiric treatment of suspected typhoid cases is common, typically using relatively inexpensive antibiotics. ${ }^{7}$ To reduce the overuse of empiric antibiotics, the end-user cost for a typhoid POC test was set at $<$ US\$3.00 (minimum requirement) or $<\mathrm{US} \$ 1.00$ (optimum requirement) to reflect the cost of empiric antibiotics in endemic regions. ${ }^{7}$ Delphi survey feedback indicated that the highest cost to the end-user in Africa should be equivalent to US $\$ 1$.

\section{DISCUSSION}

Typhoid diagnostic tests currently lack the sensitivity and specificity required for an accurate diagnosis at the POC, resulting in the overuse of antibiotics through empiric treatment. The WHO has developed a list of characteristics that make a test suitable for the resource-limited settings where typhoid is prevalent: the ASSURED acronym stands for affordable, sensitive, specific, userfriendly, rapid and robust, equipment-free, and delivered 
to those in need. ${ }^{40}$ TPPs build on these criteria and increasingly are used in the global health community to guide development of diagnostic tests and to inform donors about global health priorities. ${ }^{28} 3839$ This TPP outlines the minimum and optimum desired characteristics for an improved typhoid RDT and is intended to accelerate development of optimised diagnostics that meet the needs of users in endemic regions.

Antibiotic resistance is a growing threat to typhoid treatment, with strains of $S$. Typhi that are resistant to three first-line agents now prevalent in parts of Asia and Africa. ${ }^{8}$ The emergence of a multidrug-resistant clone in Sindh, Pakistan that has acquired additional resistance to fluoroquinolones and third-generation cephalosporins, known as extensively drug-resistant $S$. Typhi, has left azithromycin and the costly intravenous carbapenem drugs as the only antibiotic options for some patients. ${ }^{8}$ There have been sporadic case reports of azithromycin-resistant $S$. Typhi, ${ }^{8}$ but if extremely drug-resistant strains acquire azithromycin resistance, carbapenems could be left as the only effective treatment. To prevent the further spread of resistant $S$. Typhi it would be beneficial to conduct drug susceptibility testing for individual patients before commencing antibiotic therapy. Drug susceptibility testing was not included as a TPP requirement because it is not likely to be feasible in non-culture POC tests due to the evolving nature of typhoid resistance ${ }^{8}$ and may make interpretation of test results too complex for users at the lowest healthcare level. ${ }^{31}$ However, some Delphi survey respondents felt that for an RDT ever to replace blood culture it must include susceptibility testing. An RDT for diagnosis combined with epidemiological knowledge of the antibiotic sensitivity of strains, updated at intervals, could be a compromise solution.

The 2017 Global Burden of Disease study estimated that $S$. Paratyphi affected 3.4 million people annually, with 19.1 thousand deaths, compared with 10.9 million cases and 116.8 thousand deaths for $S$. Typhi. ${ }^{2}$ The higher morbidity and mortality of $S$. Typhi makes it a greater public health concern, but the increasing prevalence of $S$. Paratyphi in certain regions makes it prudent for a next-generation test to detect $S$. Paratyphi as well as $S$. Typhi. ${ }^{511} 42$ Delphi survey feedback noted that not being able to detect $S$. Paratyphi could undermine clinician confidence in a next-generation typhoid POC test as $S$. Typhi and $S$. Paratyphi may cause indistinguishable clinical syndromes. ${ }^{4}$ As drug-resistant typhoid continues to spread, it may become necessary to differentiate between these two serovars prior to starting therapy due to different antibiotic susceptibility profiles. ${ }^{43}$

The minimum diagnostic requirement for a typhoid RDT in this TPP was $\geq 90 \%$ sensitivity and $\geq 95 \%$ specificity, with an optimum threshold of $\geq 95 \%$ sensitivity and $\geq 98 \%$ specificity. Consensus agreement was achieved in the Delphi survey for specificity but not sensitivity, reflecting the substantial variability seen in the published literature with proposed targets ranging from 80\%-90\% for sensitivity and 90\%-98\% for specificity. ${ }^{23} 3637$ The poor sensitivity of blood culture as a reference standard for typhoid diagnosis makes it difficult to accurately assess the performance of novel diagnostic tests. ${ }^{33}$ Diagnostic accuracy can be improved by reliably identifying alternative infections and by using a CRS that incorporates the results of multiple tests. ${ }^{7}$ CRSs that have been used for typhoid diagnosis include culture and/or clinical findings; culture and/or Widal test; culture and/ or PCR; culture, Widal test and/or clinical finding; and culture, PCR and/or Widal test. ${ }^{33}$ Evaluation of CRSs has been limited by a lack of consistency between studies, with a systematic review identifying 21 studies that stated use of a CRS as the reference method for a typhoid diagnostic test, but no 2 studies utilised an identical CRS. For a CRS to be reproducible, it has been proposed that the individual test components need to be standardised, with clinical signs that match the WHO definition of suspected typhoid fever, assays that are commercially available and well-defined blood culture parameters. ${ }^{33}$ Further work is needed to identify a standardised CRS before it can be included in a typhoid TPP.

Typhoid fever is transmitted by the faeco-oral route in water and food contaminated by $S$. Typhi in human faeces and so is endemic in low-resource environments that lack access to clean water and adequate sanitation. While improvements in the infrastructure for water, sanitation and hygiene could reduce or eliminate typhoid, these are costly long-term endeavours. The newly approved Typbar-TCV vaccine may help to reduce the global burden of enteric fever caused by $S$. Typhi, ${ }^{44}$ but an improved diagnostic test is required to accurately estimate disease incidence and facilitate targeted vaccine deployment. To have a meaningful impact on the overuse of antibiotics that has contributed to the emergence of resistance in $S$. Typhi and other bacteria, an improved typhoid POC test needs to be used as part of a treatment algorithm in conjunction with diagnostics for malaria and other causes of acute febrile illness. The isolated use of a disease-specific diagnostic test for a febrile patient may help focus treatment if positive, but a negative test may result in alternative empiric antibiotic therapy, as seen for malaria. ${ }^{26}{ }^{45}$ Drug susceptibility testing for $S$. Typhi, performed at reference laboratories, could inform local treatment algorithms based on regional antibiotic susceptibilities. ${ }^{46}$

This work provides the first comprehensive TPP for a next-generation POC test for typhoid fever. The main limitations of this study were the lack of consensus agreement for all TPP characteristics, and the relatively low-response rate in the second round of the Delphi survey. The length of the survey may have been a barrier to completion due to the amount of time required to provide feedback on all 35 TPP characteristics. Further discussion among the typhoid community is needed to settle on the optimal target level of the healthcare system and the required diagnostic sensitivity. While this TPP is a first-step towards improved awareness of the typhoid diagnostic needs, it is crucial to keep the conversation 
going and to engage global health funders, diagnostics developers and national policymakers in the discussion on how improved diagnostic tools and related innovations can be used to improve surveillance data as well as support patient management decisions in the context of universal healthcare.

Acknowledgements We thank all the participants of the two rounds of Delphi surveys for their invaluable contribution to shape the TPP and highlight open questions. We would further like to thank the UK aid from the British people for supporting this work at FIND.

Contributors RGM, HH and SD contributed to the initial study design including the literature review and development of the draft TPP. CMP provided foundational work that formed part of the draft TPP. RGM and SD conducted the Delphi survey. CMP participated in the Delphi survey and provided key comments for shaping the final TPP. The manuscript was conceived and written by RGM and SD with input from $\mathrm{HH}$ and CMP. All authors participated in revising the manuscript and approved the final version.

Funding This study was funded by Department for International Development (http://dx.doi.org/10.13039/501100000278).

Competing interests None declared.

Patient consent for publication Not required.

Provenance and peer review Not commissioned; externally peer reviewed.

Data availability statement All data relevant to the study are included in the article or uploaded as supplementary information.

Open access This is an open access article distributed in accordance with the Creative Commons Attribution Non Commercial (CC BY-NC 4.0) license, which permits others to distribute, remix, adapt, build upon this work non-commercially, and license their derivative works on different terms, provided the original work is properly cited, appropriate credit is given, any changes made indicated, and the use is non-commercial. See: http://creativecommons.org/licenses/by-nc/4.0/.

\section{ORCID iDs}

Richard G Mather http://orcid.org/0000-0003-0893-4513

Heidi Hopkins http://orcid.org/0000-0003-1076-6758

Christopher M Parry http://orcid.org/0000-0001-7563-7282

Sabine Dittrich https://orcid.org/0000-0002-4522-2788

\section{REFERENCES}

1 MacFadden DR, Bogoch II, Andrews JR. Advances in diagnosis, treatment, and prevention of invasive Salmonella infections. Curr Opin Infect Dis 2016;29:453-8.

2 GBD 2017 Disease and Injury Incidence and Prevalence Collaborators. Global, regional, and national incidence, prevalence, and years lived with disability for 354 diseases and injuries for 195 countries and territories, 1990-2017: a systematic analysis for the global burden of disease study 2017. Lancet 2018;392:1789-858.

3 Dicker D, Nguyen G, Abate D, et al. Global, regional, and national age-sex-specific mortality and life expectancy, 1950-2017: a systematic analysis for the global burden of disease study 2017. Lancet 2018;392:1684-735.

4. Maskey AP, Day JN, Phung QT, et al. Salmonella enterica serovar paratyphi $\mathrm{A}$ and $\mathrm{S}$. enterica serovar typhi cause indistinguishable clinical syndromes in Kathmandu, Nepal. Clin Infect Dis 2006:42:1247-53.

5 Bhutta ZA. Current concepts in the diagnosis and treatment of typhoid fever. BMJ 2006;333:78-82.

6 Parry CM, Wijedoru L, Arjyal A, et al. The utility of diagnostic tests for enteric fever in endemic locations. Expert Rev Anti Infect Ther 2011;9:711-25.

7 Andrews JR, Ryan ET. Diagnostics for invasive Salmonella infections: current challenges and future directions. Vaccine 2015;33:C8-15.

$8 \mathrm{Klemm}$ EJ, Shakoor S, Page AJ, et al. Emergence of an extensively drug-resistant Salmonella enterica serovar typhi clone harboring a promiscuous plasmid encoding resistance to fluoroquinolones and third-generation cephalosporins. MBio 2018;9:e00105-18.

9 Basnyat B. Typhoid versus typhus fever in post-earthquake Nepal. Lancet Glob Health 2016;4:e516-7.

10 Schroeder LF, Elbireer A, Jackson JB, et al. Laboratory Diagnostics Market in East Africa : A Survey of Test Types, Test Availability, and Test Prices in Kampala, Uganda. PLoS Negl Trop Dis 2015:10:e0134578.

11 Raffatellu M, Wilson RP, Winter SE, et al. Clinical pathogenesis of typhoid fever. J Infect Dev Ctries 2008;2:260-6.

12 Baker S, Favorov M, Dougan G. Searching for the elusive typhoid diagnostic. BMC Infect Dis 2010;10:45.

13 Wain J, Diep TS, Ho VA, et al. Quantitation of bacteria in blood of typhoid fever patients and relationship between counts and clinica features, transmissibility, and antibiotic resistance. J Clin Microbiol 1998:36:1683-7.

14 Khanam F, Sheikh A, Sayeed MA, et al. Evaluation of a Typhoid/ Paratyphoid diagnostic assay (TPTest) detecting anti-Salmonella IgA in secretions of peripheral blood lymphocytes in patients in Dhaka, Bangladesh. PLoS Negl Trop Dis 2013;7:e2316.

15 World Health Organization. Typhoid vaccines: WHO position paper March 2018. Geneva, 2018. Available: http://www.who.int/wer

16 von Kalckreuth V, Konings F, Aaby P, et al. The typhoid fever surveillance in Africa program (TSAP): clinical, diagnostic, and epidemiological methodologies. Clin Infect Dis 2016;62:S9-16.

17 Tulip Diagnostics. Tydal widal antigen set. Available: http://www. tulipgroup.com/Tulip_New/html/pack_inserts/Tydal.pdf [Accessed 20 Aug 2018].

18 IDL Biotech AB. TUBEX TF rapid typhoid detection. Available: http:// idlbiotech.com/wp-content/uploads/2018/08/IDL_TUBEX_folder1511-web.pdf [Accessed 16 Aug 2018].

19 Reszon Diagnostics. Typhidot rapid IgG/IgM (Combo) version 2, 2014. Available: https://www.reszonics.com/wp-content/uploads/ 2017/04/Reszon-IFU-TYPHIDOT-Rapid-IgG_IgM-combo-2014-04. pdf [Accessed 16 Aug 2018].

20 LifeAssay Diagnostics. Test-itTM typhoid IgM lateral flow assay, 2016. Available: https://www.viaglobalhealth.com/wp-content/ uploads/2016/03/Test-it-Typhoid-Instructions.pdf [Accessed 16 Aug 2018].

21 Das S, Rajendran K, Dutta P, et al. Validation of a new serologybased dipstick test for rapid diagnosis of typhoid fever. Diagn Microbiol Infect Dis 2013;76:5-9.

22 World Health Organization Communicable Disease Surveillance and Response Vaccines and Biologicals. Background document: the diagnosis, treatment and prevention of typhoid fever. Geneva, 2003. Available: http://www.who.int/rpc/TFGuideWHO.pdf

23 Wijedoru L, Mallett S, Parry CM. Rapid diagnostic tests for typhoid and paratyphoid (enteric) fever. Cochrane Database Syst Rev 2017;5.

24 Islam K, Sayeed MA, Hossen E, et al. Comparison of the performance of the TPTest, Tubex, Typhidot and widal immunodiagnostic assays and blood cultures in detecting patients with typhoid fever in Bangladesh, including using a Bayesian latent class modeling approach. PLoS Negl Trop Dis 2016;10:e0004558.

25 Felgner J, Jain A, Nakajima R, et al. Development of ELISAs for diagnosis of acute typhoid fever in Nigerian children. PLoS Negl Trop Dis 2017;11:e0005679.

26 Bruxvoort KJ, Leurent B, Chandler CIR, et al. The impact of introducing malaria rapid diagnostic tests on fever case management: a synthesis of ten studies from the act Consortium. Am J Trop Med Hyg 2017;97:1170-9.

27 Marks F, von Kalckreuth V, Aaby P, et al. Incidence of invasive Salmonella disease in sub-Saharan Africa: a multicentre populationbased surveillance study. The Lancet Global Health 2017;5:e310-23.

28 Dittrich S, Tadesse BT, Moussy F, et al. Target product profile for a diagnostic assay to differentiate between bacterial and non-bacterial infections and reduce antimicrobial overuse in resource-limited settings: an expert consensus. PLoS One 2016;11:e0161721-12.

29 Denkinger CM, Dolinger D, Schito M, et al. Target product profile of a molecular drug-susceptibility test for use in microscopy centers. $J$ Infect Dis 2015;211:S39-49.

30 Das JK, Hasan R, Zafar A, et al. Trends, associations, and antimicrobial resistance of Salmonella typhi and paratyphi in Pakistan. Am J Trop Med Hyg 2018;99:48-54.

31 Ghani AC, Burgess DH, Reynolds A, et al. Expanding the role of diagnostic and prognostic tools for infectious diseases in resourcepoor settings. Nature 2015;528:S50-2.

32 Mogasale V, Ramani E, Mogasale VV, et al. What proportion of Salmonella typhi cases are detected by blood culture? A systematic literature review. Ann Clin Microbiol Antimicrob 2016;15:32.

33 Storey HL, Huang Y, Crudder C, et al. A meta-analysis of typhoid diagnostic accuracy studies: a recommendation to adopt a standardized composite reference. PLoS One 2015;10:e0142364

34 Obaro SK, Iroh Tam P-Y, Mintz ED, et al. The unrecognized burden of typhoid fever. Expert Rev Vaccines 2017;16:249-60.

35 Thriemer K, Ley B, Menten J, et al. A systematic review and metaanalysis of the performance of two point of care typhoid fever 
tests, Tubex TF and Typhidot, in endemic countries. PLoS One 2013;8:e81263.

36 Maude RR, de Jong HK, Wijedoru L, et al. The diagnostic accuracy of three rapid diagnostic tests for typhoid fever at Chittagong medical college Hospital, Chittagong, Bangladesh. Trop Med Int Health 2015;20:1376-84.

37 Andrews JR, Arora P, Bogoch II, et al. Understanding the potential value of new diagnostics for enteric fever : insights from decision analytic modeling. Am J Trop Med Hyg 2017;97.

38 Ding XC, Ade MP, Baird JK, et al. Defining the next generation of Plasmodium vivax diagnostic tests for control and elimination: target product profiles. PLoS Negl Trop Dis 2017;11:e0005516-15.

39 Chua AC, Cunningham J, Moussy F, et al. The case for improved diagnostic tools to control Ebola virus disease in West Africa and how to get there. PLoS Negl Trop Dis 2015;9:e0003734.

40 Urdea M, Penny LA, Olmsted SS, et al. Requirements for high impact diagnostics in the developing world. Nature 2006;444:73-9.

41 Radhakrishnan A, Als D, Mintz ED, et al. Introductory article on global burden and epidemiology of typhoid fever. Am J Trop Med Hyg 2018;99:4-9.

42 Darton TC, Blohmke CJ, Pollard AJ. Typhoid epidemiology, diagnostics and the human challenge model. Curr Opin Gastroenterol 2014;30:7-17.

43 McKinnon LR, Karim QA. Host-Pathogen interactions: honing in on enteric fever. Elife 2014;3:e03545.
44 Neuzil KM, Pollard AJ, Marfin AA. Introduction of typhoid conjugate vaccines in Africa and Asia. Clin Infect Dis 2019;68:S27-30.

45 Hopkins H, Bruxvoort KJ, Cairns ME, et al. Impact of introduction of rapid diagnostic tests for malaria on antibiotic prescribing: analysis of observational and randomised studies in public and private healthcare settings. BMJ 2017;356.

46 Parry CM, Ribeiro I, Walia K, et al. Multidrug resistant enteric fever in South Asia: unmet medical needs and opportunities. BMJ 2019;37.

47 Steele AD, Hay Burgess DC, Diaz Z, et al. Challenges and opportunities for typhoid fever control: a call for coordinated action. Clin Infect Dis 2016;62:S4-8.

48 Shao AF, Rambaud-Althaus C, Samaka J, et al. New algorithm for managing childhood illness using mobile technology (ALMANACH): a controlled non-inferiority study on clinical outcome and antibiotic use in Tanzania. PLoS One 2015;10:e0132316.

$49 \mathrm{Kim}$ J-Y, Ji S-Y, Goo Y-K, et al. Comparison of rapid diagnostic tests for the detection of Plasmodium vivax malaria in South Korea. PLoS One 2013;8:64353

50 World Health Organization. Laboratory biosafety manual - Third edition. Geneva, 2004. Available: http://www.who.int/csr/resources/ publications/biosafety/Biosafety7.pdf?ua=1

51 World Health Organization. Risk based classification of diagnostics for who prequalification, 2014. Available: http://www.who.int/ diagnostics_laboratory/evaluations/140513_who_risk_based classification_of_ivds_for_pq_buffet.pdf [Accessed 1 Aug 2018]. 